\title{
Vacuum Analysis of Texas Foil Model 6 Serial 7
}

David B. Augustine

Fermilab

October 26, 2009 


\section{Scope:}

The purpose of this document is to describe the testing and analysis of Texas Foil Model 6, Serial 7 All future references to this device in this document will be known as TX6\#7. This device did not meet operational vacuum of 1E-8 Torr or better.

\section{History:}

This device has been in storage at PAB for 3 years at atmosphere. It was capped to keep the dust out. It was brought out of storage in July of 2009 to reference survey, and installation. TX6\#7 was set up to bake at MI-60, at $100^{\circ} \mathrm{C}$. The University of Texas, at Austin Texas, provided a bake out procedure specifying the temperature. The best pressure obtained cold was $1 \mathrm{E}-6$ Torr. The best pressure obtained after $100^{\circ} \mathrm{C}$ bake was 3E-7 Torr. The installation was stopped based upon this measurement because the device would not meet what is considered operational vacuum.

Test: 
TX6\#7 was instrumented, pumped out, leak checked, and set up to bake by 9-18-09. It was leak checked with a sensitivity of $1.5 \mathrm{E}-10$ torr*1/s. Figure 1 shows an overview of the vacuum equipment attached for testing. On the left side of the picture are a $20 \mathrm{l} / \mathrm{s}$ ion pump, ion gauge, and pump out port. All valves are all-metal type. There is another allmetal valve installed on the ion pump to isolate the pump during an outgassing measurement. This is the ion pump manifold. On the right side of the picture is a similar manifold with an RGA. This is the RGA manifold. Each manifold can be independently isolated from TX6\#7 with all metal valves. Note that this RGA only works on faraday cup mode. Each manifold was independently pumped and baked at $150^{\circ} \mathrm{C}$. The foil can was baked at $100^{\circ} \mathrm{C}$. The effective pumping speed on the TX6\#7 from the ion pump manifold was calculated to be 7 l/s. Prior to evacuation and bake, the TX6\#7 was tested for motor drive and electrical signal functions. This test was repeated when the vacuum testing was completed.

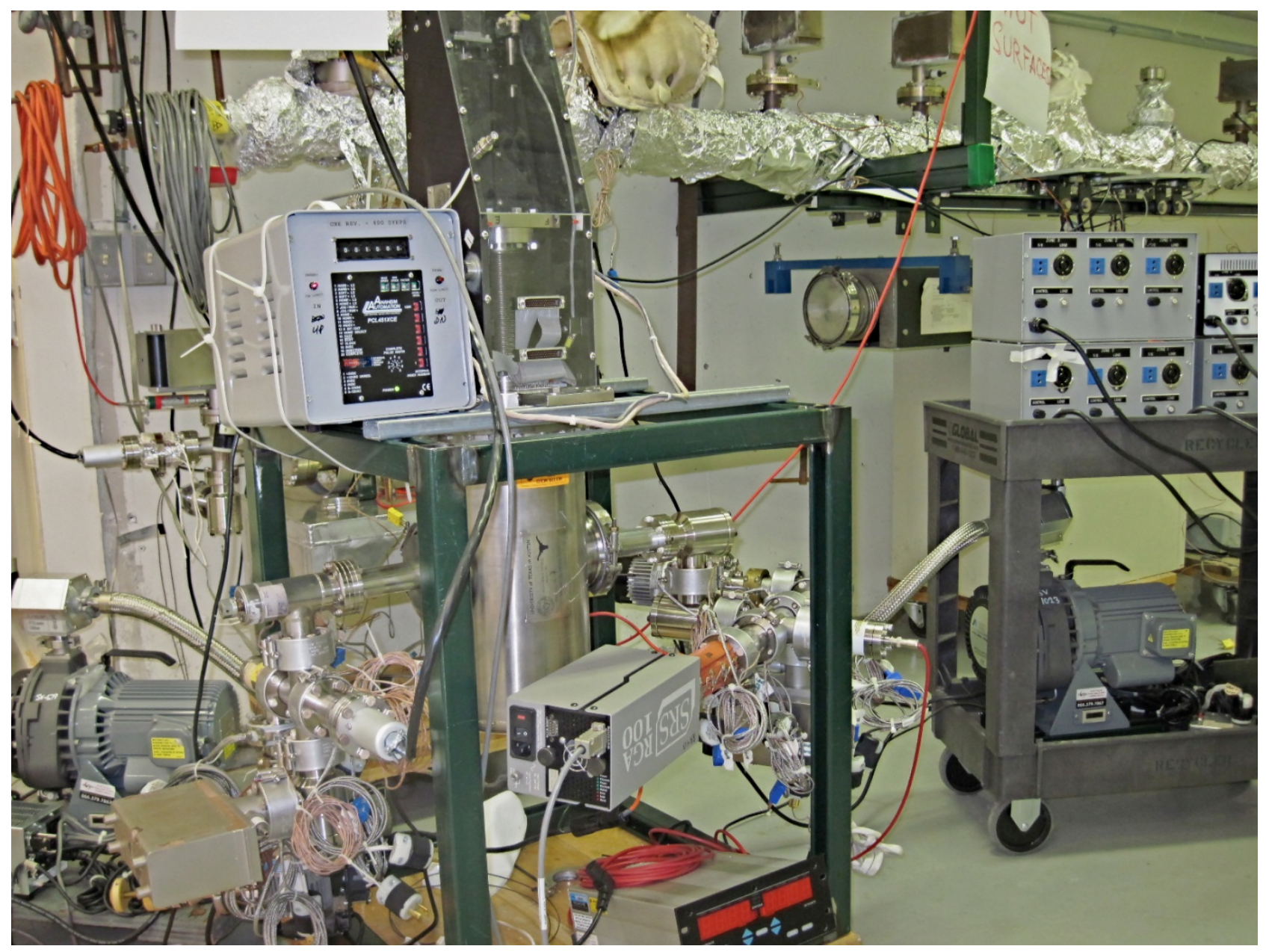

Figure 1. Test setup for vacuum analysis of the TX6\#7. 
The residual gas analysis graph shown in Figure 2 is the baseline scan for the RGA manifold. This was done after the $150^{\circ} \mathrm{C}$ bake. The filament was on for 20 hours before the scan was taken.

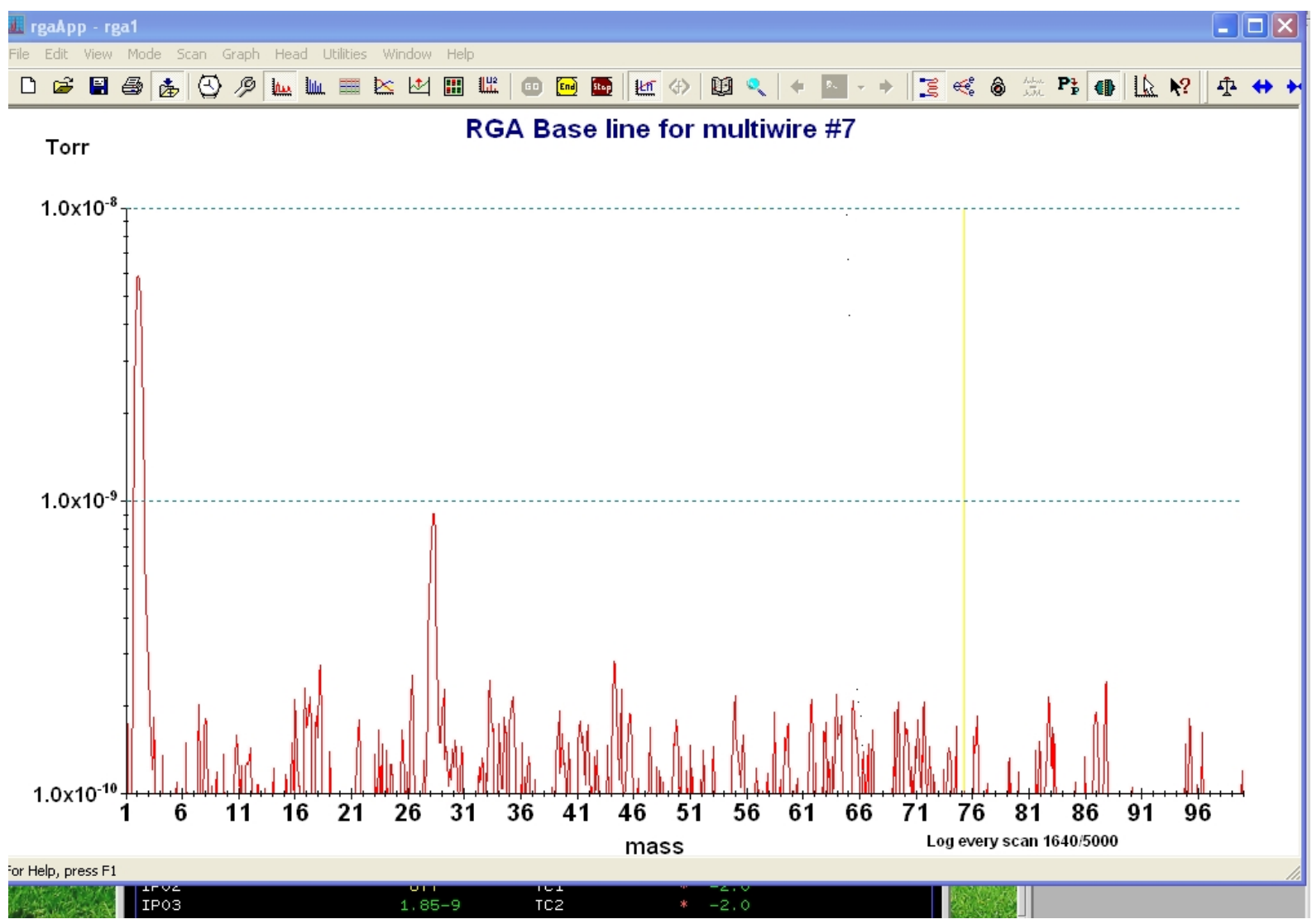

Figure 2. RGA manifold baseline scan. 
Figure 3 shows the graph of the bake out and vent test. The bake was started at approximately 11 am Friday, 9-18-09. The bake was terminated when the pressure flat lined at approximately 8 am Monday, 9-21-09. As TX6\#7 cooled, the pressure fell to 8E9 Torr. Note that at the best pressure two $20 \mathrm{l} / \mathrm{s}$ ion pumps were on at the same time. When the RGA manifold ion pump was isolated, the pressure rose to approximately 1.5E-8 Torr.

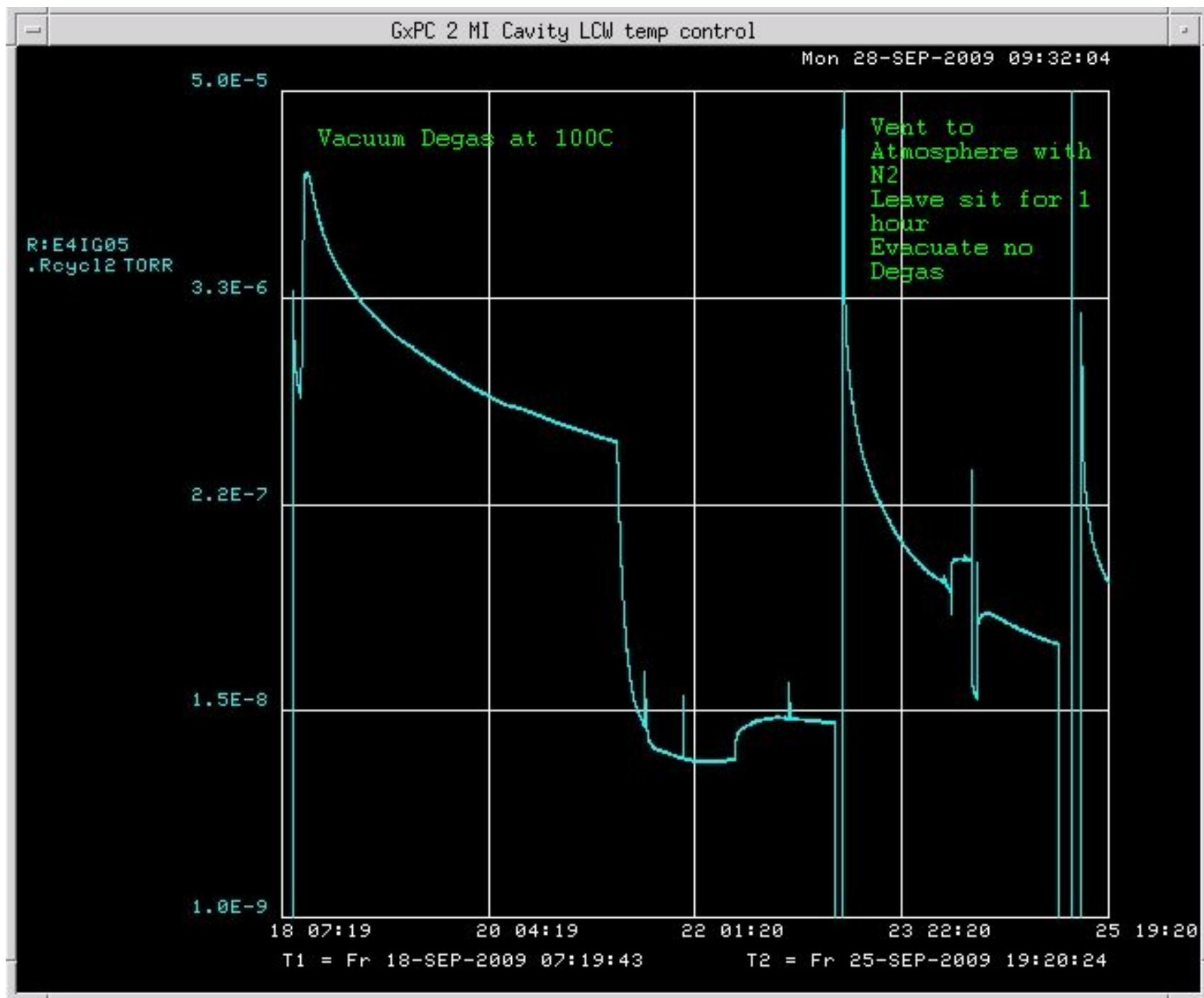

Figure 3. Pumpdown curve for the bakeout and vent test. 
Figure 4 shows the RGA scan taken prior to baking TX6\#7 at $100^{\circ} \mathrm{C}$. The scan shows that it contains adsorbed atmospheric gas. Although leak tested and determined to be helium leak tight at 1.2E-10 torr l/s, the scan shows peaks at 32 (oxygen) and 40 (argon). Peak 12 (carbon) is greater than peak 14 (nitrogen), therefore peak 28 is carbon monoxide. This indicates that no air leak was present, as was confirmed when the vacuum chamber was leak checked with this same RGA head.

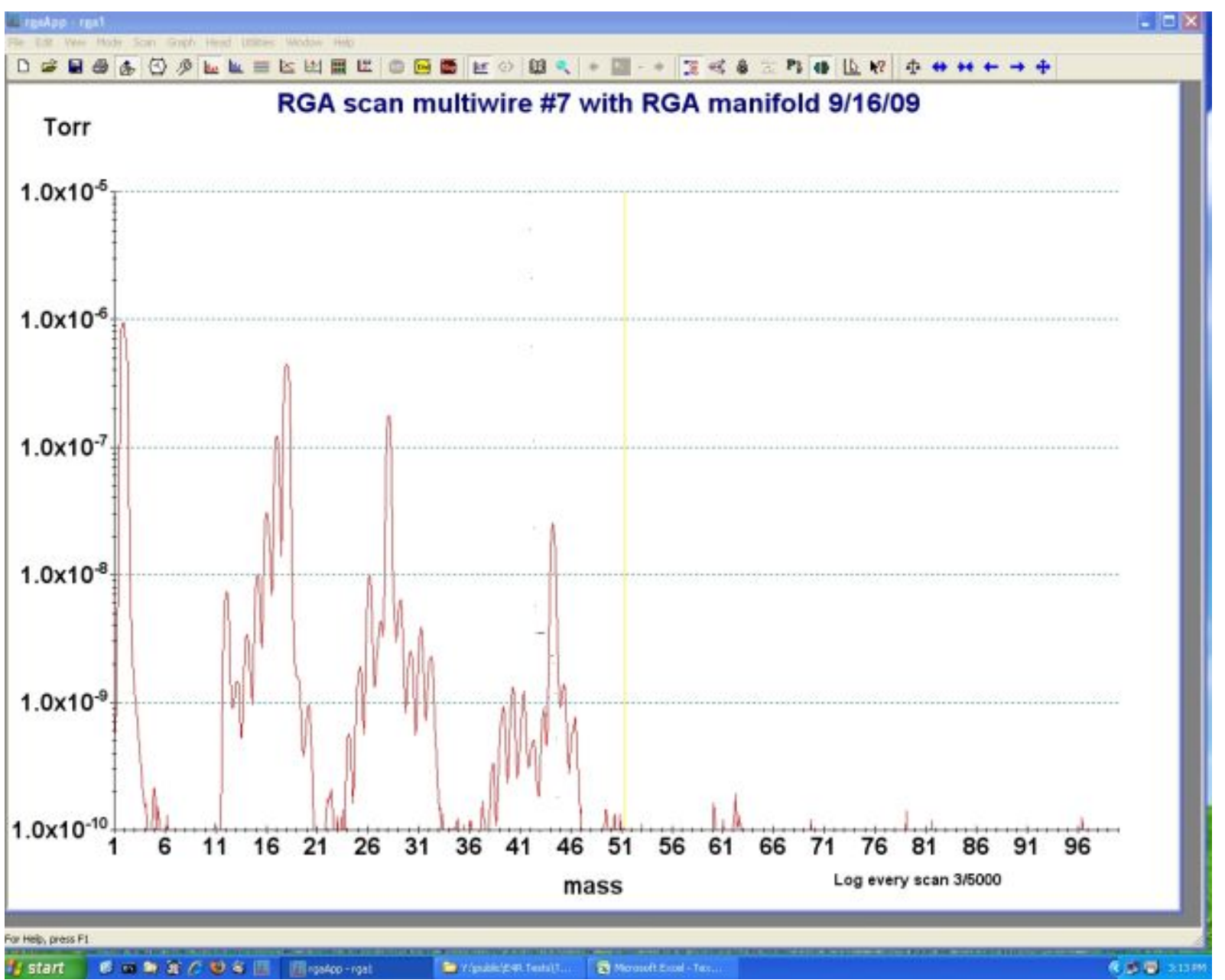

Figure 4. RGA scan of TX6\#7 prior to bake. 
The RGA scan in Figure 5 was taken 24 hours after the $100^{\circ} \mathrm{C}$ bake. All gases show a marked reduction, especially argon and oxygen.

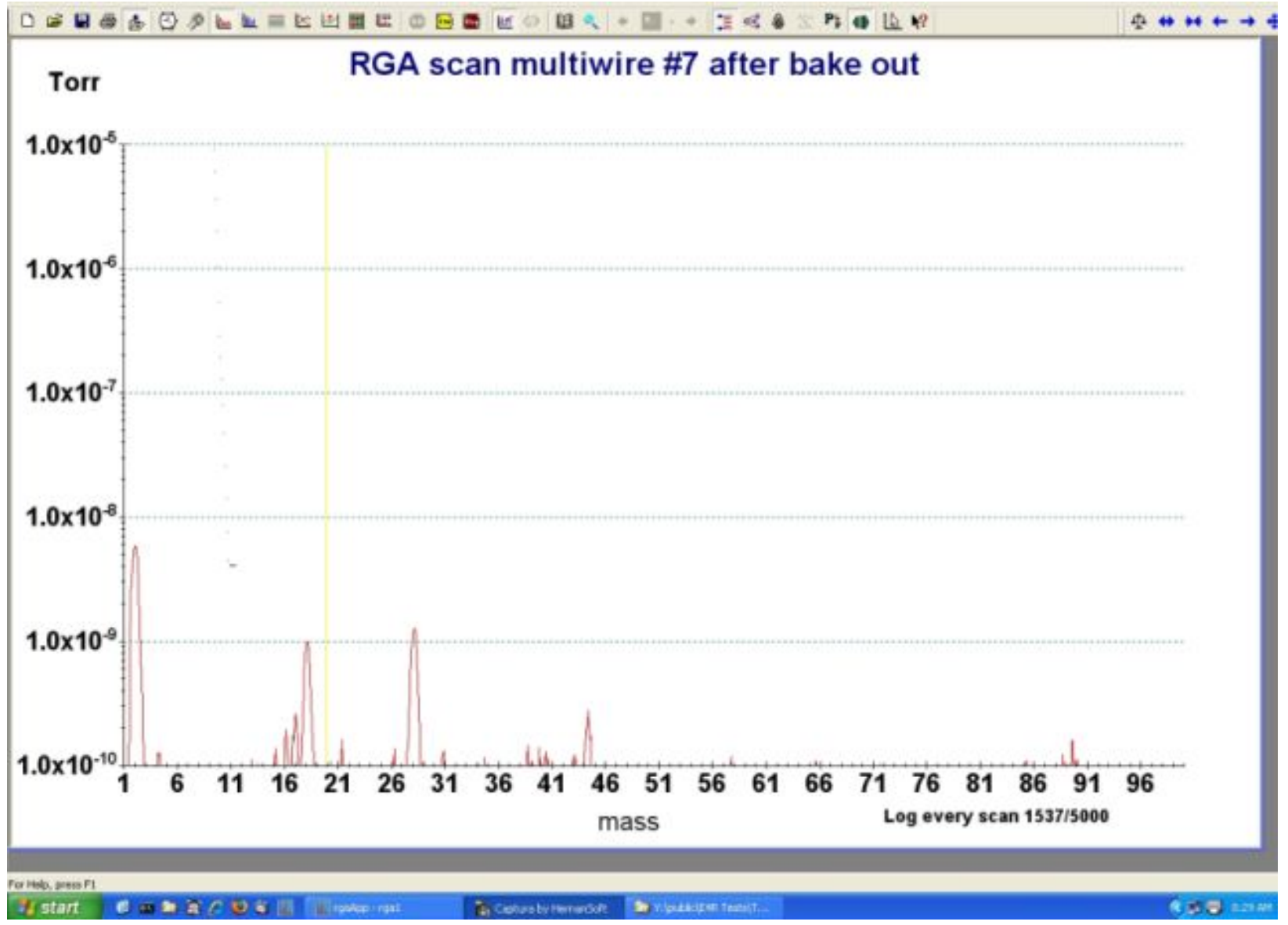

Figure 5. RGA scan of the TX6\#7 foil taken 24 hours after the $100^{\circ} \mathrm{C}$ bakeout. 


\section{Vent to Atmosphere with Nitrogen Gas:}

All pumps and gauges were turned off or isolated from the ion pump manifold. The RGA manifold ion pump was left on as was the RGA. The valve between this manifold and TX6\#7 was closed. The vacuum was vented to atmosphere and held there for one hour. The TX6\#7 was then pumped out and ion pump turned on. Once vacuum was stable, another RGA scan was performed (Figure 6).

It is important to note that all gases rose about a factor of ten in approximately the same ratio as the scan performed prior to the bake. Argon and oxygen did not return. Due to the defective electron multiplier, it is difficult to determine the 28 peak at this level of resolution.

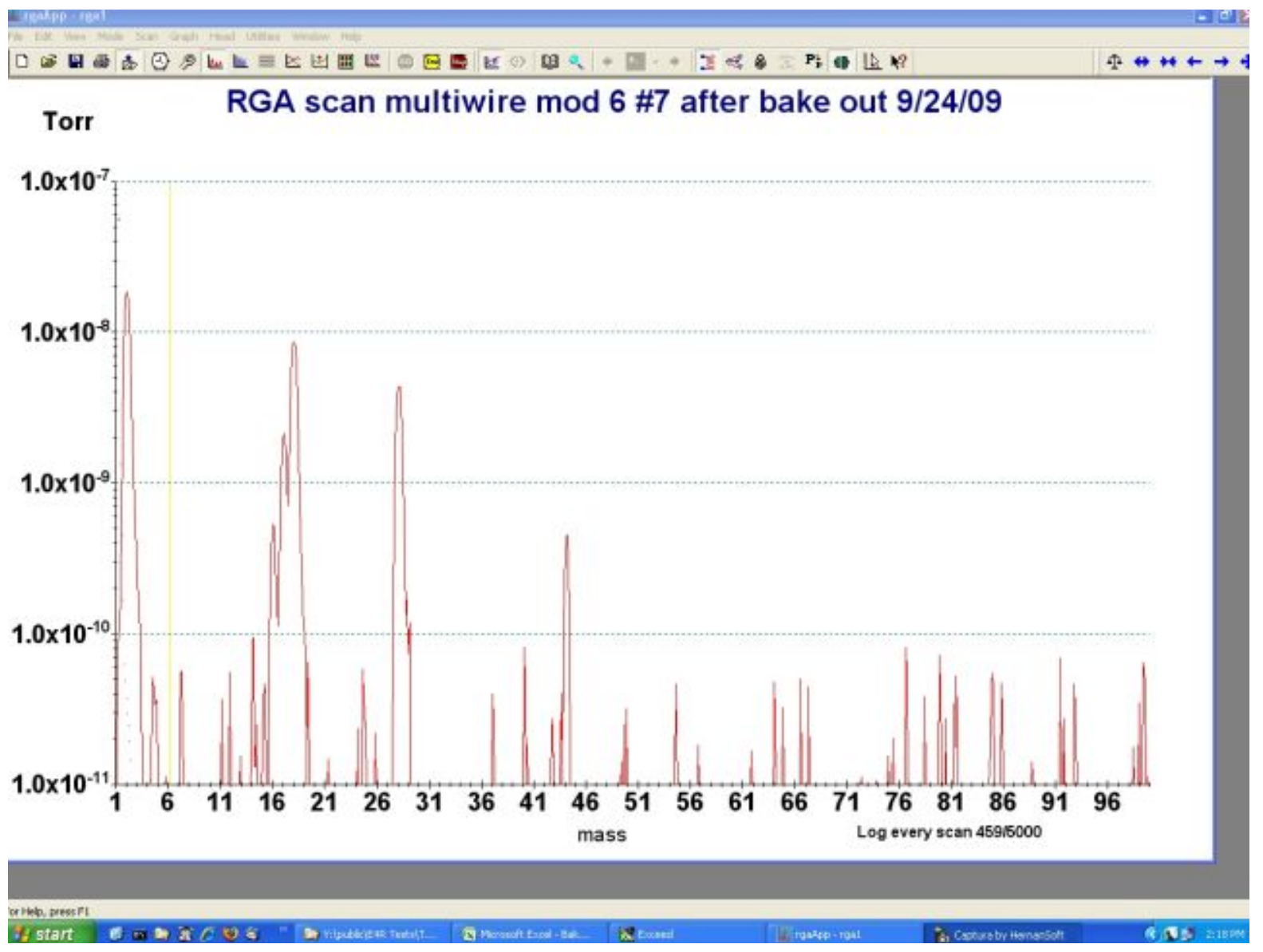

Figure 6. RGA scan of TX6\#7 after vent to atmosphere.

\section{Notes:}


The TX6\#7 was vented to atmosphere with nitrogen. It was left idle for one hour. Then the TX6\#7 was pumped back out and the ion pump turned on. The vacuum returned to 3 E-8 Torr overnight. This test was to mimic the installation in the tunnel. The effective pumping speed on a comparable Texas foil installation in the MI $8 \mathrm{GeV}$ line is calculated at $25 \mathrm{l} / \mathrm{s}$, which is three times better than the effective pumping speed of the test setup. The TX6\#7 should recover to a 10-8 Torr vacuum within a couple days without baking. The TX6\#7 will have some small exposure to atmospheric air during the installation process. It is imperative that it is continuously purged with dry nitrogen during the installation process.

\section{Conclusion:}

Based upon the testing above, there is material in this device that readily absorbs atmospheric gases, especially water. This device was designed and built by the University of Texas, at Austin Texas. We do not have exact construction details. However, the construction of this device is similar to Fermi-designed foil monitors. Based on this experience, it is likely that the problem material is the epoxy used to bond the foil substrate to the ceramic plane. It appears that if the device is, baked in advance, kept clean and stored under vacuum prior to installation, and is continuously purged with N2 while the device is being surveyed and connected, then it should achieve low E-8 Torr vacuum within a few days after installation without a recovery bake.

\section{Installation Guidelines}

In order to keep this device fit for installation the following things must be done:

- Keep this device actively pumped with an ion pump when in storage.

- This device is sensitive to mechanical shock. The foils are thin and closely spaced. The vibration of movement on hard wheels, rapid vent up to atmosphere or rapid pump down could cause the foil strips to touch one another and electrically short. Use pneumatic tired vehicles. Do not jar in transport or installation

- Prior to installation, vent the beam tube and flow nitrogen from both directions if possible, then vent the device to atmosphere.

- Immediately install the device into the beam tube that has nitrogen flowing through it. Rough align the flanges enough that they can be taped or wrapped with plastic to contain the nitrogen purge.

- Connect vacuum flanges as soon as possible.

- Evacuate as soon as possible. 
All tests were performed at E4R. The RGA data and this document is stored at \\Beamssrv1\mechsupt.bd\public\E4R TEST

Technicians: Rom Bacino, Fernando Juarez, Dan Schoo, Linda Purcell-Taylor, Advisors: Ron Kellett, Lucy Nobrega, Linda Valerio 